\title{
Resonant wind-driven oceanic motions
}

\author{
Anne-Laure Dalibard ${ }^{\mathrm{a}, \mathrm{b}}$, Laure Saint-Raymond ${ }^{\mathrm{c}}$ \\ a Université Paris-Dauphine, Ceremade, F-75016 Paris, France \\ ${ }^{\mathrm{b}} C N R S, U M R 7534$, F-75016 Paris, France \\ ${ }^{\mathrm{c}}$ École normale supérieure, DMA - UMR CNRS 8553, F-75005 Paris, France \\ Received *****; accepted after revision +++++ \\ Presented by ééééé
}

\begin{abstract}
We are interested here in describing the linear response of a rapidly rotating fluid to some surface stress, possibly due to the wind. The distinctive feature of the model considered here lies in the fact that the stress admits fast time oscillations and may be resonant with the Coriolis force. In addition to the usual Ekman layer, we exhibit another - much larger - boundary layer, and some global vertical profile. We prove in particular that for large times, the wind effect is no longer localized in the vicinity of the surface.
\end{abstract}

\section{Résumé}

\section{Forçage résonnant de fluides océaniques}

Cette note est consacrée à la description des effets d'un forçage surfacique, par exemple dû au vent, sur des fluides en rotation rapide dont l'évolution est régie par une équation linéaire. La particularité de l'analyse menée ici réside dans le caractère fortement oscillant en temps du vent, qui peut alors entrer en résonance avec la force de Coriolis. En particulier, la taille des couches limites dûes au forçage résonnant est beaucoup plus grande que celle des couches d'Ekman habituelles, et il apparaît un profil vertical singulier, qui, en temps grand, n'est pas localisé dans un voisinage de la surface.

\section{Version française abrégée}

Dans cette note, on étudie le comportement asymptotique d'un système linéaire de type Navier-StokesCoriolis, qui modélise l'évolution des courants océaniques; précisément, on considère la solution $u$ du système

Email addresses: dalibard@ceremade.dauphine.fr (Anne-Laure Dalibard), Laure.Saint-Raymond@ens.fr (Laure Saint-Raymond). 


$$
\begin{array}{r}
\partial_{t} u+\frac{1}{\epsilon} e_{3} \wedge u-\Delta_{h} u-\nu \partial_{z z} u+\nabla p=0, \quad t>0, x_{h} \in \mathbf{T}^{2}, z \in[0,1] \\
\nabla \cdot u=0
\end{array}
$$

muni des conditions aux bords

$$
\begin{aligned}
& u_{\mid z=0}=0, \quad u_{3 \mid z=1}=0, \quad \partial_{z} u_{h \mid z=1}\left(t, x_{h}\right)=\beta \sigma\left(\frac{t}{\epsilon}, x_{h}\right), \\
& u_{\mid t=0}=\gamma,
\end{aligned}
$$

où $\beta>0, \mathbf{T}^{2}=(\mathbf{R} / 2 \pi \mathbf{Z})^{2}$, et où le forçage (modélisé par $\sigma$ ) est une fonction régulière, presque périodique en temps et $\mathbf{T}^{2}$-périodique en $x_{h}$.

On s'intéresse ici à l'influence du vent, plus particulièrement lorsqu'il présente des oscillations rapides à des fréquences égales à celle de la rotation de la Terre. On montre alors que le forçage $\sigma$, s'il est résonnant, génère des couches limites de taille bien supérieure à celle des couches d'Ekman habituelles. De plus, pour des profils particuliers de la fonction $\sigma$, l'ensemble du fluide est déstabilisé en temps grand (voir [2]).

Une analyse multi-échelles formelle montre que l'opérateur de Coriolis $L$, défini par

$$
L: u \mapsto e_{3} \wedge u+\nabla p, \quad \text { où } p \text { vérifie }\left\{\begin{array}{l}
\operatorname{div}\left(e_{3} \wedge u+\nabla p\right)=0, \\
\partial_{z} p_{\mid z=0}=\partial_{z} p_{\mid z=1}=0,
\end{array}\right.
$$

engendre des oscillations temporelles à des fréquences d'ordre $\epsilon^{-1}$. On s'attend donc à ce qu'il existe une fonction $\bar{u}(t)$ telle que la solution $u$ du système linéaire ci-dessus se comporte comme $\exp (-t L / \epsilon) \bar{u}(t)$.

Le résultat principal est le suivant :

Théorème $\mathbf{0 . 1}$ On suppose que les hypothèses (3)-(4) sont vérifiées. Soit $\gamma \in L^{2}\left(\mathbf{T}^{2} \times[0,1]\right)$, à divergence nulle et flux nul. Soit $u \in \mathcal{C}\left(\mathbf{R}^{+}, L^{2}\left(\mathbf{T}^{2} \times[0,1]\right)\right) \cap L_{l o c}^{2}\left(\mathbf{R}^{+}, H^{1}\left(\mathbf{T}^{2} \times[0,1]\right)\right)$ l'unique solution du système linéaire, et soit $\bar{u} \in \mathcal{C}\left(\mathbf{R}^{+}, L^{2}\left(\mathbf{T}^{2} \times[0,1]\right)\right) \cap L^{2}\left(\mathbf{R}_{+}, H_{h}^{1}\left(\mathbf{T}^{2} \times[0,1]\right)\right)$ la solution du système

$$
\begin{array}{r}
\partial_{t} \bar{u}-\Delta_{h} \bar{u}+\sqrt{\frac{\nu}{\epsilon}} S_{\text {Ekman }}(\bar{u})=0, \quad \operatorname{div} \bar{u}=0, \\
\bar{u}_{\mid t=0}=\gamma, \quad \bar{u}_{3 \mid z=0}=\bar{u}_{3 \mid z=1}=0,
\end{array}
$$

où $S_{E k m a n}$ est un opérateur linéaire, continu, positif sur le sous-espace de $L^{2}\left(\mathbf{T}^{2} \times[0,1]\right)$ constitué des fonctions à divergence et flux nuls (voir [1] pour une définition précise). Alors lorsque $\epsilon, \nu \rightarrow 0$,

$$
u(t)-\exp \left(-\frac{t}{\epsilon} L\right) \bar{u}(t) \rightarrow 0 \quad \text { dans } L_{l o c}^{\infty}\left(\mathbf{R}^{+}, L^{2}\right) \cap L_{l o c}^{2}\left(\mathbf{R}^{+}, H_{h}^{1}\right) .
$$

La preuve de ce résultat repose sur une méthode d'énergie classique (voir [1]), qui permet d'estimer la différence entre la fonction $u$ et une solution approchée explicite : cette approximation est obtenue comme la somme du terme principal $\exp (-t L / \epsilon) \bar{u}(t)$, de termes de couches limites, et de termes correctifs intérieurs. Le rôle de ces termes correcteurs, petits en norme $L^{2}$, est de faire en sorte que la solution approchée satisfasse le système linéaire avec des termes d'erreur suffisamment petits pour obtenir de la convergence forte.

\section{Introduction}

This note is devoted to the asymptotic analysis as $\epsilon, \nu \rightarrow 0$ of the linear system

$$
\begin{array}{r}
\partial_{t} u+\frac{1}{\epsilon} e_{3} \wedge u-\Delta_{h} u-\nu \partial_{z z} u+\nabla p=0, \quad t>0, x_{h} \in \mathbf{T}^{2}, z \in[0,1], \\
\nabla \cdot u=0,
\end{array}
$$


supplemented with the boundary conditions

$$
\begin{aligned}
& u_{\mid z=0}=0, \quad u_{3 \mid z=1}=0, \quad \partial_{z} u_{h \mid z=1}\left(t, x_{h}\right)=\beta \sigma\left(\frac{t}{\epsilon}, x_{h}\right), \\
& u_{\mid t=0}=\gamma,
\end{aligned}
$$

where $\beta$ is a positive number, $\sigma:=\sigma\left(\tau, x_{h}\right)$ is some smooth function, periodic in $x_{h}$ and almost periodic in $\tau$, and $\mathbf{T}^{2}=[0,2 \pi)^{2}$ denotes the two-dimensional torus.

This system is a linear version of the Navier-Stokes-Coriolis equations, which describe the motion of oceanic currents. Here, we focus on the influence of the wind-stress $\sigma$ on the global oceanic circulation. More precisely, we are interested in the effects of a resonant forcing, i.e. of a wind oscillating with the same period as the rotation of the Earth. In contrast with the non-resonant case, which was studied by Desjardins and Grenier [3] and then by Masmoudi [5], we prove that the forcing on resonant modes creates boundary layers which are much larger than the usual ones, and may furthermore destabilize the whole fluid inside the domain for large times.

\section{Main results and strategy}

A formal multiscale analysis of (1) shows that the Coriolis operator $L$, defined by $L:=\mathbb{P}\left(e_{3} \wedge \cdot\right)$, where $\mathbb{P}$ is the orthogonal projection of $L^{2}\left(\mathbf{T}^{2} \times[0,1]\right)$ onto the subspace

$$
V_{0}:=\left\{u \in L^{2}\left(\mathbf{T}^{2} \times[0,1]\right) \quad / \quad \nabla \cdot u=0 \text { and } u_{3 \mid z=0}=u_{3 \mid z=1}=0\right\},
$$

generates fast time oscillations, at frequencies of order $1 / \epsilon$. More precisely, one can prove (see for instance [1]) that there exists a hilbertian basis of $V_{0}$, denoted by $\left(N_{k}\right)_{k \in \mathbf{Z}^{3} \backslash\{0\}}$, constituted of eigenvectors of the linear penalization: for all $k \in \mathbf{Z}^{3} \backslash\{0\}$, we have

$$
L N_{k}=\mathbb{P}\left(e_{3} \wedge N_{k}\right)=i \lambda_{k} N_{k}, \quad \text { where } \lambda_{k}=-\frac{k_{3} \pi}{\sqrt{\left|k_{h}\right|^{2}+\left(\pi k_{3}\right)^{2}}} .
$$

We therefore expect $u$ to behave like some function $\exp (-t L / \epsilon) \bar{u}(t)$.

In order to understand the evolution with respect to the slow time variable, the idea is then to get rid of the penalization term by filtering out the oscillations in equation (1) (see $[4,6]$ ), that is, by composing equation (1) by the Coriolis semi-group $\exp (t L / \epsilon)$. The filtered function $u_{L}(t):=\exp (t L / \epsilon) u(t)$ satisfies a linear equation with vanishing viscosity (and without any penalization term); passing to the limit in the latter yields the so-called 'envelope equation' (see (5) below).

Assumptions on the wind-stress $\sigma$. Without any loss of generality, since the equation is linear, we will assume that $\sigma$ has a finite number of Fourier modes, i.e.

$$
\exists M \subset \mathbf{R},|M|<\infty, \exists N \in \mathbf{N}, \forall\left(\tau, x_{h}\right), \quad \sigma\left(\tau, x_{h}\right)=\sum_{\mu \in M} \sum_{\substack{k_{h} \in \mathbf{Z}^{2},\left|k_{h}\right| \leq N}} \hat{\sigma}\left(\mu, k_{h}\right) e^{i \mu \tau} e^{i k_{h} \cdot x_{h}} .
$$

We further require that the strength of the wind-stress is not too large, namely

$$
\exists\left(\alpha_{0}, \alpha_{1}\right) \in \mathbf{R}^{2}, \quad \alpha_{0}<7 / 12, \alpha_{1}>1 / 4, \quad \beta=O\left(\nu^{-\alpha_{0}} \epsilon^{\alpha_{1}}\right) .
$$

Then the following result holds:

Theorem 2.1 Let $\gamma \in V_{0}$, and let $u \in \mathcal{C}\left(\mathbf{R}^{+}, V_{0}\right) \cap L_{\text {loc }}^{2}\left(\mathbf{R}^{+}, H^{1}\left(\mathbf{T}^{2} \times[0,1]\right)\right)$ be the unique solution of (1)-(2). Let $\bar{u} \in \mathcal{C}\left(\mathbf{R}^{+}, V_{0}\right) \cap L^{2}\left(\mathbf{R}_{+}, H_{h}^{1}\left(\mathbf{T}^{2} \times[0,1]\right)\right)$ be given by 


$$
\begin{aligned}
\partial_{t} \bar{u}-\Delta_{h} \bar{u}+\sqrt{\frac{\nu}{\epsilon}} S_{E k m a n}(\bar{u}) & =0, \\
\bar{u}_{\mid t=0} & =\gamma,
\end{aligned}
$$

where the operator $S_{E k m a n}: V_{0} \rightarrow V_{0}$ is linear, positive and continuous (see [1] for a precise definition).

Then under assumptions (3), (4), as $\epsilon, \nu \rightarrow 0$,

$$
u(t)-\exp \left(-\frac{t}{\epsilon} L\right) \bar{u}(t) \rightarrow 0
$$

in $L_{l o c}^{\infty}\left(\mathbf{R}^{+}, L^{2}\left(\mathbf{T}^{2} \times[0,1]\right)\right) \cap L_{l o c}^{2}\left(\mathbf{R}^{+}, L^{2}\left(\mathbf{T}^{2} \times[0,1]\right)\right)$.

Remark 1 (i) The above theorem holds for all values of the ratio $\epsilon / \nu$, but the asymptotic behaviour of $\bar{u}$ depends on the relative values of $\epsilon, \nu$. In particular, if $\nu / \epsilon \rightarrow \infty$, then all modes such that $k_{h} \neq 0$ in $\bar{u}$ vanish as $\exp \left(-c_{k} t \sqrt{\nu / \epsilon}\right)$, due to the positivity of the operator $S_{E k m a n}$.

(ii) If the forcing $\sigma$ bears on resonant modes only, namely if

$$
\sigma\left(\tau, x_{h}\right)=\hat{\sigma}^{+} e^{i \tau}(1, i)+\hat{\sigma}^{-} e^{-i \tau}(1,-i),
$$

then we are able to prove a global result: in this case, let

$$
u^{\text {sing }}\left(t, x_{h}, z\right)=\frac{\beta}{2} \sum_{ \pm} \sum_{k_{3} \in 2 \mathbf{Z}+1}\left(\frac{2}{k_{3} \pi}\right)^{2}(-1)^{\frac{k_{3}-1}{2}} \hat{\sigma}^{ \pm} \sin \left(\frac{\pi}{2} k_{3} z\right) e^{ \pm i \frac{t}{\epsilon}}\left[1-e^{-\nu k_{3}^{2} t}\right](1, \pm i, 0) .
$$

Then

$$
u(t)-\left[\exp \left(-\frac{t}{\epsilon} L\right) \bar{u}(t)+u^{\operatorname{sing}}(t)\right] \rightarrow 0
$$

in $L^{\infty}\left(\mathbf{R}^{+}, L^{2}\left(\mathbf{T}^{2} \times[0,1]\right)\right) \cap L^{2}\left(\mathbf{R}^{+}, L^{2}\left(\mathbf{T}^{2} \times[0,1]\right)\right)$. In particular, for large times, $u(t) \approx u^{\text {sing }}(t) \sim \beta$. Since $\beta$ may be very large, there is a destabilization of the whole fluid inside the domain as $t \rightarrow \infty$.

(iii) Notice that the two convergences (6) and (8) are compatible, since with assumption (4), $u^{\text {sing }}=$ $O\left(\nu^{3 / 4} \beta\right)=o(1)$ in $L^{2}\left([0, T] \times \mathbf{T}^{2} \times[0,1]\right), \forall T>0$.

\section{Elements of proof}

The strategy of proof follows that developed in [1]: since we intend to prove a strong convergence result, the idea is to construct an approximate solution, which is the sum of the principal term $\exp (-t L / \epsilon) \bar{u}(t)$, of boundary layer terms and of corrector interior terms, and which satisfies the system (1)-(2) with sufficiently small error terms. The convergence then follows from a standard energy method.

Notice that the linearity of the equation allows us to use a superposition principle, so that it is sufficient to prove Theorem 2.1 for the solution $u^{\text {bottom }}$ of (1)-(2) with $u_{\mid t=0}^{\text {bottom }}=\gamma, \partial_{z} u_{h \mid z=1}^{\text {bottom }}=0$ on the one hand, and for the solution $u^{\text {top }}$ of (1)-(2) with $u_{\mid t=0}^{\text {top }}=0, \partial_{z} u_{h \mid z=1}^{\text {top }}=\beta \sigma$ on the other. Concerning the term $u^{\text {bottom }}$, the proof of convergence can be found in $[1,5]$; in this case, the main point lies in the construction of boundary layer terms, which restore the horizontal boundary conditions violated by $\exp (-t L / \epsilon) \bar{u}(t)$ at $z=0$. The typical size of the boundary layers is then $\sqrt{\epsilon \nu}$.

Thus the study of the asympotic behaviour of $u^{t o p}$ is the main novelty of this work. We only sketch the main steps of the proof here, and we refer to [2] for details. Note that because $u_{\mid z=0}^{t o p}=0$, we expect the main term of the approximation to be zero. Let us also emphasize that there is no Ekman pumping term due to the wind in the envelope equation (5) because of assumption (4): indeed, the amplitude of the Ekman pumping is $\nu \beta$, which is $o(1)$ in our case. Therefore, we only have to build boundary and interior correctors. 


\subsection{The boundary layer and the singular profile}

First, we define a boundary layer term $u^{B L}$ which matches the horizontal boundary conditions at $z=1$, and which takes the form

$$
u^{B L}\left(t, x_{h}, z\right)=\beta \sqrt{\epsilon \nu} \sum_{\mu \in M} \sum_{k_{h}} \sum_{ \pm} \lambda_{ \pm}\left(\mu, k_{h}\right)^{-1} \exp \left(i \frac{\mu t}{\epsilon}+i k_{h} \cdot x_{h}-\frac{(1-z) \lambda_{ \pm}\left(\mu, k_{h}\right)}{\sqrt{\epsilon \nu}}\right) \hat{u}_{ \pm}^{B L}\left(\mu, k_{h}\right),
$$

where $\Re\left(\lambda_{ \pm}\left(\mu, k_{h}\right)\right)>0$ and $\hat{u}_{ \pm}^{B L}\left(\mu, k_{h}\right)$, to be chosen later, is $O(1)$ and such that $\operatorname{div} u^{B L}=0$. Inserting this expression into $(1)$, we infer that the decay rate $\lambda_{ \pm}\left(\mu, k_{h}\right)$ is obtained as a solution of the equation

$$
\operatorname{det}\left(\begin{array}{cc}
i \mu-\lambda^{2}+\epsilon k_{h}^{2}+\frac{\epsilon \nu k_{1} k_{2}}{\lambda^{2}-\epsilon \nu k_{h}^{2}} & -1-\frac{\epsilon \nu k_{1}^{2}}{\lambda^{2}-\epsilon \nu k_{h}^{2}} \\
1+\frac{\epsilon \nu k_{2}^{2}}{\lambda^{2}-\epsilon \nu k_{h}^{2}} & i \mu-\lambda^{2}+\epsilon k_{h}^{2}-\frac{\epsilon \nu k_{1} k_{2}}{\lambda^{2}-\epsilon \nu k_{h}^{2}}
\end{array}\right)=0 .
$$

Explicit calculations then lead to the following results:

- If $|\mu| \neq 1$, we infer that $\lambda\left(\mu, k_{h}\right)$ is bounded away from zero.

- If $|\mu|=1$ and $k_{h} \neq 0$, we prove that there exists a constant $C \geq 1$ such that

$$
C^{-1}\left[(\epsilon \nu)^{1 / 4}+\sqrt{\epsilon}\right] \leq\left|\lambda\left(\mu, k_{h}\right)\right| \leq C\left[(\epsilon \nu)^{1 / 4}+\sqrt{\epsilon}\right] .
$$

As a consequence, the size of the boundary layer for these 'quasi-resonant modes' is of order $(\epsilon \nu)^{1 / 4}$, which is much larger than the size of the classical Ekman layers.

- If $|\mu|=1$ and $k_{h}=0$, a thorough analysis shows that the boundary condition cannot be absorbed entirely in some boundary layer, no matter how large. Hence, our treatment of these modes is slighlty different, and we define a function $u^{\text {stat }}$ by

$$
u^{\text {stat }}\left(t, x_{h}, z\right)=\frac{\beta}{2} z \sum_{ \pm}\left(\hat{\sigma}_{1}( \pm 1,0) \mp i \hat{\sigma}_{2}( \pm 1,0)\right) \exp \left( \pm i \frac{t}{\epsilon}\right)(1, \pm i, 0) .
$$

Since $u_{\mid t=0}^{\text {stat }}$ does not vanish, we consider the solution $u^{o s c}$ of (1), supplemented with the initial condition $u_{\mid t=0}^{o s c}=-u_{\mid t=0}^{s t a t}$, together with homogeneous boundary conditions at $z=0$ and $z=1$; the singular term $u^{\text {sing }}$ is then obtained as the sum of $u^{\text {stat }}$ and $u^{o s c}$. Moreover, $\exp (t L / \epsilon) u^{o s c}$ satisfies a heat equation with homogeneous boundary conditions, so that explicit calculations are tractable in Fourier space, and lead to expression (7).

\subsection{The interior terms and the equation for the mean motion}

In the second step, we take into account the vertical boundary conditions at $z=0$ and $z=1$. Notice that in general, the divergence-free property prevents $u^{B L}$ from satisfying the no-flux boundary conditions. Precisely, the third component of $u^{B L}$ is of order $\beta(\epsilon \nu)^{\frac{1}{4}}$ in $L^{2}\left(\mathbf{T}^{2}\right)$. Thus, an interior corrector $u^{i n t}$ is introduced, which restores the Dirichlet boundary condition on the vertical component, and which satisfies the evolution equation up to error terms which are $o(1)$ in $L^{2}$. The explicit construction is as follows: we set $u^{\text {int }}=v^{\text {int }}+\delta u^{i n t}$, where

$$
\begin{array}{r}
v_{3}^{i n t}\left(t, x_{h}, z\right)=-z u_{3}^{B L}\left(t, x_{h}, 1\right), \\
v_{h}^{i n t}\left(t, x_{h}, z\right)=\nabla_{h} \Delta_{h}^{-1} u_{3}^{B L}\left(t, x_{h}, 1\right),
\end{array}
$$

and 


$$
\begin{array}{r}
\partial_{t} \delta u^{i n t}+\frac{1}{\epsilon} \mathbb{P} \delta u^{i n t}-\Delta_{h} \delta u^{i n t}=-\mathbb{P}_{K}\left[\partial_{t} v^{i n t}+\frac{1}{\epsilon} e_{3} \wedge v^{i n t}-\Delta_{h} v^{i n t}\right] \\
\delta u_{\mid t=0}^{i n t}=0,
\end{array}
$$

where $\mathbb{P}_{K}$ is the $L^{2}$ projection on the vector space generated by $\left\{N_{k},|k| \leq K\right\}$. The truncation parameter $K$ is chosen so that $u^{\text {int }}$ is a solution of equation (1) with a small error term, and the term $\delta u^{\text {int }}$ is small in $H^{s}$ norm for some $s>3 / 2$. A careful analysis shows that $K$ depends on $\epsilon$ and $\nu$; this dependance ultimately prescribes the scaling condition (4).

When constructing the approximate solution for $u^{\text {bottom }}$, the envelope equation (5) is obtained precisely at this step, by filtering the part of $v^{\text {int }}$ which is resonant with the main term $\exp (-t L / \epsilon) \bar{u}(t)$.

\subsection{Iterating the approximation process}

The horizontal component of the interior term $u^{\text {int }}$ constructed above does not vanish at $z=0$; whence, other boundary layer terms, of lower order than $u^{B L}$, must be defined so as to restore the horizontal boundary conditions. In fact, constructing merely one additional boundary layer term is not sufficient to jump to a conclusion, and we must apply several times the two first steps described above. After a few iterations, the remaining boundary terms are of order $o(\epsilon)$ in $H^{s}\left(\mathbf{T}^{2}\right)$ for all $s$, which allows us to complete the proof of convergence thanks to the following Lemma:

Lemma 3.1 (Stopping condition) Let $\delta_{0}, \delta_{1} \in L^{\infty}\left(\mathbf{R}^{+}, H^{3}\left(\mathbf{T}^{2}\right)\right)$ be two families such that $\int_{\mathbf{T}^{2}} \delta_{i, 3}=0$ and $\left\|\delta_{i}\right\|_{L^{\infty}\left(\mathbf{R}^{+}, H^{3}\left(\mathbf{T}^{2}\right)\right)}=o(\epsilon),\left\|\partial_{t} \delta_{i}\right\|_{L^{\infty}\left(\mathbf{R}^{+}, H^{3}\left(\mathbf{T}^{2}\right)\right)}=o(1)$.

Then there exists a family $w \in L^{\infty}\left(\mathbf{R}^{+}, L^{2}\left(\mathbf{T}^{2} \times[0,1]\right)\right)$ with $\nabla \cdot w=0$ such that

$$
w_{\mid z=0}=\delta_{0}, \quad w_{3 \mid z=1}=\delta_{1,3} \text { and } \partial_{z} w_{h \mid z=1}=\delta_{1, h}
$$

and satisfying the following estimates

$$
\|w\|_{L^{2}\left(\mathbf{T}^{2} \times[0,1]\right)} \rightarrow 0 \text { and }\left\|\partial_{t} w+\frac{1}{\epsilon} L w-\nu \partial_{z z} w-\Delta_{h} w\right\|_{L^{2}\left(\mathbf{T}^{2} \times[0,1]\right)} \rightarrow 0 \text { as } \epsilon \rightarrow 0 .
$$

\section{Acknowledgements}

The first author wishes to thank the Département de mathématiques et applications (École normale supérieure, Paris), for its hospitality during the preparation of this work.

\section{References}

[1] J.-Y. Chemin, B. Desjardins, I. Gallagher and E. Grenier, Basics of Mathematical Geophysics, Oxford Lecture Series in Mathematics and its Applications, 32 (2006), Oxford University Press.

[2] A.-L. Dalibard and L. Saint-Raymond. Mathematical study of resonant wind-driven oceanic motions, preprint (2008).

[3] B. Desjardins and E. Grenier, On the Homogeneous Model of Wind-Driven Ocean Circulation, SIAM Journal on Applied Mathematics, 60 (1999), 43-60.

[4] E. Grenier, Oscillatory perturbations of the Navier-Stokes equations. Journal de Mathématiques Pures et Appliquées, 76 (1997), 477-498.

[5] N. Masmoudi, Ekman layers of rotating fluids: the case of general initial data, Communications in Pure and Applied Mathematics, 53 (2000), 432-483.

[6] S. Schochet, Fast singular limits of hyperbolic PDEs. Journal of Differential Equations, 114 (1994), 476-512. 